\title{
The Occasional Maverick of Analytic Tradecraft
}

\author{
David R. Mandel
}

Defence Research and Development Canada, Toronto, Canada

David R. Mandel

Defence Research and Development Canada

Toronto Research Centre

1133 Sheppard Avenue West

Toronto, Ontario

M3K 2C9

drmande166@gmail.com

(416) 635-2000 ext. 3146

David R. Mandel is a senior Defence Scientist with Defence Research and Development Canada and Adjunct Professor of Psychology at York University. He publishes widely in peer-reviewed journals and has five co-authored or co-edited books on the topics of reasoning, judgment, and decision-making. Mandel is Chairman of the NATO System Analysis and Studies Panel Research Technical Group on Assessment and Communication of Uncertainty in Intelligence to Support Decision Making (SAS-114) and Principal Investigator of multiple Canadian government projects aimed at improving intelligence production through the application of decision science.

Acknowledgement: This work was supported by Canadian Safety and Security Program project CSSP-2018-TI-2394. I thank Daniel Irwin for his feedback on an earlier draft of this article and his assistance with its preparation.

Words: 4,570 
The Occasional Maverick of Analytic Tradecraft

Roughly a decade ago, in a commissioned report I wrote for the US National Research Council's Committee on Behavioral and Social Science Research to Improve Intelligence Analysis for National Security, I characterized those rare and seminal contributors to analytic tradecraft—figures such as Sherman Kent, Jack Davis, and Richards Heuer - as mavericks. ${ }^{1}$ The term was meant to conjure a heroic image: mavericks are like mythical forces of good bursting upon an otherwise dreary set. They possess energy, brains and noble motives and act to save their communities while others around them are silent or worse. Notwithstanding those noble characteristics, I made the case for why intelligence communities should not choose or be forced to rely on the occasional maverick.

The following year, my argument that intelligence communities exhibited overreliance on the occasional maverick was summarized in a more widely circulated workshop report by the National Research Council's Planning Committee on Field Evaluation of Behavioral and Cognitive Sciences-Based Methods and Tools for Intelligence and Counterintelligence. ${ }^{2}$ Here, I update my views on the occasional maverick using as a jumping-off point a recent article in this journal by Stephen Couthart that explores the case history of one of the intelligence community's greatest mavericks, Richards Heuer $\mathrm{Jr}^{3}$

The intelligence maverick is often idealized but seldom described for what this occasional figure in intelligence history truly represents: a symptom of longstanding organizational neglect when it comes to developing sound analytic methods for the production and dissemination of intelligence. Indeed, Coulthart's analysis provides a case in point. ${ }^{4}$ He portrays Heuer as the figure who, against all odds, rescues cognitive psychology for intelligence tradecraft from oblivion. Where Daniel Kahneman and Zvi 
Lanir failed, Heuer succeeds, proving that all is not lost on policymakers who "just don't get it." Coulthart successfully highlights the importance of fortuitous encounters and timing, but his analysis misses critical factors that complete both the case history of Heuer and the more general case of the occasional maverick.

I agree with Coulthart that Heuer was an effective knowledge integrator. $\mathrm{He}$ successfully integrated early academic research on heuristics and biases into the intelligence community through bias awareness training and the development of structured analytic techniques (SATs) that have since become mandated elements of US intelligence policy for promoting analytic integrity. ${ }^{5}$ However, Coulthart bypasses whether any of these efforts were ultimately effective and why the intelligence community did not have a more proactive approach to leveraging science for the good of intelligence production. The fact that Heuer's influence on analytic training and tradecraft has spread over the past two decades since the release of his book does not speak directly to the effectiveness question. ${ }^{6}$ To address that question, research and skeptical analysis is needed. For instance, do SATs, such as Analysis of Competing Hypotheses (ACH), which the US and allied intelligence communities expect their analysts to use, actually improve the soundness of reasoning and judgment accuracy? ${ }^{7}$

In principle, there are good reasons to doubt that SATs work effectively. One reason to doubt the effectiveness of the SAT approach is that it shows virtually no concern for how the multi-stage assessment processes required by most SATs might ramp up noise production - a form of disregard Welton Chang, Elissabeth Berdini, Philip Tetlock and I call noise neglect. ${ }^{8}$ For example, what does it mean in ACH to assess the consistency between hypothesis and evidence? This could be interpreted as the probability of the evidence given the hypothesis, the probability of the hypothesis given the evidence, or an intuitive judgment of the degree to which the evidence 
matches the hypothesis to name some possibilities (If it is the latter, then $\mathrm{ACH}$ may in fact be increasing the probability of cognitive bias by correspondingly increasing the probability that analysts apply the bias-promoting representativeness heuristic as a routine part of their hypothesis-testing process! ${ }^{9}$ )

A second reason for skepticism is that the conceptualization of cognitive bias underpinning the SAT approach is invariably unipolar, even though most cognitive biases are bipolar. ${ }^{10}$ For example, analysts might be miscalibrated by being overconfident or by being underconfident, yet scant attention has been paid to the latter bias. Without knowing where along a bias spectrum an analyst's (or analytic unit's) behavior lies it is impossible to know what form of debiasing may be required. A SAT aimed at reducing overconfidence might inadvertently increase underconfidence. This is hardly far-fetched. Alan Barnes and I found that intelligence assessments produced over a roughly 6-year period by a strategic intelligence unit he directed were significantly underconfident. ${ }^{11}$ Warning such analysts about the perils of overconfidence probably does not improve their calibration and could make it worse, and yet this is precisely what analytic training tends to do. ${ }^{12}$ Likewise, as Stephen Marrin has noted, analytic training teaches SATs even though these methods have had little scientific testing to determine their effectiveness. ${ }^{13}$

Recent studies of $\mathrm{ACH}$ also challenge the assumption that the method improves judgment. In one experiment, intelligence analysts who were randomly assigned to an $\mathrm{ACH}$ or no-SAT control group were required to assess the probabilities of four alternative hypotheses on the basis of several diagnostic pieces of evidence. ${ }^{14}$ Compared to analysts in the control group, analysts in the ACH group were significantly less coherent and marginally less accurate. Analysts in the ACH group were also less likely to utilize relevant base-rate information. ${ }^{15}$ The deleterious effects of $\mathrm{ACH}$ on judgment 
quality were observed in another recent experiment involving three mutually exclusive hypotheses that recruited a larger sample of participants. ${ }^{16}$ Yet another study recently published in this journal found no evidence that $\mathrm{ACH}$ improved judgment accuracy or mitigated confirmation bias or serial position effects. ${ }^{17}$

In addition to mounting evidence that $\mathrm{ACH}$ is not effective and perhaps even harmful to good judgment, there are important conceptual challenges that are specific to $\mathrm{ACH}$ (unlike the earlier two — noise neglect and bias polarity — that apply to most SATs). Notably, ACH mistakenly advises analysts to put aside hypothesis-supporting evidence and to focus instead on disconfirmatory evidence only when estimating the relative likelihood of alternative hypotheses. Apparently, Heuer got this idea from Sir Karl Popper's theory of falsificationism but was unaware that the notion that a single disconfirmatory instance is sufficient to disconfirm a hypothesis applies only to universal hypotheses, such as "all swans are white." 18 In fact, optimal information seeking and hypothesis test strategies depend on what form of uncertainty one aims to minimize (a function of the task environment) and on features of the information environment. ${ }^{19}$ No normative model of hypothesis testing or belief revision recommends counting up in a coarse manner the number of pieces of evidence that are judged to be inconsistent or "doubly inconsistent", doing so for each hypothesis under evaluation and ranking the relative likelihoods of alternative hypotheses on that basis.

The more general point is that even the best ideas of a few exceptional mavericks are no substitute for a program of scientific research geared towards improving analytic processes for the intelligence community. ${ }^{20}$ The occasional maverick simply cannot keep pace with relevant scientific and technological developments. Since Heuer's survey of psychology for intelligence, decades of relevant research have been conducted. Who within the intelligence community has kept it up to 
date and thought about how it could be effectively exploited? (The answer is no one.) And even if a few mavericks had kept up, would their ideas constitute a sufficient extraction of potential from that knowledge base? What is the likelihood that the best ideas that could benefit intelligence production and subsequent policy decision-making would be thought up by so few minds inculcated in the same closed community culture?

In addition to being unable to keep up with scientific developments, mavericks are also poorly positioned to test their own good ideas. This is not only because they are unlikely to be seasoned scientists with experience in devising unbiased, valid, and informative scientific tests of their proposed analytic methods, but also because there is the potential for bias even among scientists. Science requires a large enough pool of entrants that it can be left to others to disprove one's good ideas (should they prove interesting enough to draw critical attention). If there are only a few mavericks in charge of hypothesis generation, it should come as no surprise that hypothesis evaluation will suffer. Nor is evaluation bias necessarily intentional. Research shows that people are prone to exhibiting a bias blind spot in which they judge others to be more susceptible than they are to cognitive biase ${ }^{21}$ —a self-deceptive, meta-cognitive tendency that (perhaps surprisingly) appears to be unrelated to cognitive ability. ${ }^{22}$ Likewise, we tend to think our hunches are more likely to be correct than others' hunches, and therefore less in need of experimental testing.

Heuer's response to suggestions posted on an online discussion of the International Association for Intelligence Education that his $\mathrm{ACH}$ technique be empirically tested hint at such an attitude:

Can't we have confidence in making a common sense judgment that going through the process of assessing the inconsistency of evidence will generally improve the quality of analysis? Similarly, can't we have confidence in making 
a common sense judgment that starting the analysis with a set of hypotheses will, on average, lead to better analysis than starting by looking at the pros and cons for a single hypothesis? Do we really need an empirical analysis of these two points? Is it really feasible to do a high quality empirical analysis of the effectiveness of these two points? ${ }^{23}$

While Heuer's confidence in ACH's effectiveness may have exceeded the levels held by most tradecraft specialists, it is not uncommon in exchanges I have had with the latter group to encounter the argument that "while SATs may not be perfect, at least they are better than nothing." The correct response to such unbridled optimism is, but how do you know? As noted earlier, recent scientific tests of ACH show either no difference or impairment in judgment quality compared to no-SAT conditions. Yet even if we put aside these findings, is it not ironic that tradecraft experts who teach analysts à la $\mathrm{ACH}$ to list the full set of hypotheses fail to entertain one of three hypotheses that reappear virtually every time an analytic (or indeed any) method is evaluated - namely, did the method yield an improvement, no change, or a decrement in performance?

Science has been and still remains the single best antidote to overly optimistic beliefs about truth — including truth about the effectiveness of organizational methods. Tradecraft mavericks may excel as hypothesis generators, but they have not proven themselves to be competent and reliable hypothesis testers. The latter requires that they give their proposed analytic methods fair chances to fail. To do so, they must be able to design experiments that put their hypotheses to rigorous empirical tests. Experimental design is deceivingly simple, but any scientist worth his or her salt knows the simplicity is illusory. As Richard Feynman warned, panoplies of careful considerations, tradeoffs, and decisions underlie any piece of well-conducted research. ${ }^{24}$ This is not something the uninitiated can reasonably be expected to appreciate or be good at, nor should they be 
expected to be capable of adeptly analyzing experimental data and drawing sound probabilistic inferences from it. An intelligence community that relies on the emergence of mavericks to advance tradecraft, or that simply borrows what mavericks in other intelligence communities have developed without critical evaluation of methodological effectiveness, will not appear to be taking tradecraft seriously.

As William Nolte recently noted in this journal's inaugural Perspectives article, intelligence organizations, like all government organizations, are weakly motivated to change unless there is external pressure from powerful stakeholders. ${ }^{25}$ The fact that after three-quarters of a century of modern intelligence, intelligence analysts are still not given methods that have a credible body of scientific evidence to support their effectiveness is testimony to the institutional sloth that persists at the highest levels. If the capability for analysis had even half the importance of classified collections, this would not be so. ${ }^{26}$ The persistence with which the intelligence community clings to anti-scientific practices is not, as Coulthart suggests, due to the inability or unwillingness of scientists to better sell their entrepreneurial product in snappy 30second sound bites. Are the pages of journals on cryptography or geosciences more user-friendly than those describing behavioral science that could improve analytic reasoning and judgment? Of course they aren't, but a key difference is that everyone has an intuitive theory of how to reason, judge and decide and, therefore, even professionals in the business of expert judgment adamantly believe they do not need research to settle such matters.

I suspect that intelligence organizations do not face sufficient pressure from powerful policymaking elites because the latter share roughly the same beliefs. Indeed, a similar view, as Sherman Kent aptly noted, can explain why estimative intelligence is likely to be underappreciated by policymakers, despite being perhaps the most 
important type of intelligence the community could offer them. ${ }^{27}$ Policymakers endowed with the intelligence privy to analysts tend to believe they can formulate assessments and reach conclusions as effectively as analysts, or more effectively. Accordingly, estimates that align with the policymaker's conclusion prove only that the analysts can catch up to them, whereas estimates that contradict their conclusions can easily be dismissed as just a different opinion.

Granted that the US intelligence community has invested in research to improve intelligence analysis over the past decade through the Intelligence Advanced Research Programs Activity (IARPA). IARPA's programs represent an important step in moving away from reliance on mavericks to advance tradecraft. However, it remains to be seen if the intelligence community can effectively exploit IARPA-funded research findings to improve training, on-the-job support to analysts, and ultimately, the quality of finished intelligence. Will IARPA prove transformative of the anti-scientific culture that pervades the intelligence community or will it be walled off from the dominant culture?

From discussions with tradecraft specialists in the US and other NATO countries my sense is that most of IARPA's programs in support of intelligence analysis have yet to impact training or tradecraft. IARPA also funds high-risk/high-reward research and is unlikely to support careful testing of current methods - research that may not be sexy but which is sorely needed. IARPA also demands a lockstep process since bidders respond to the particulars of program requests for proposals and successful bidders are often put into competition and incentivized by a strict set of rules for winning the tournament (and subsequent years of funding). While some-notably past tournament winners ${ }^{28}$ - have touted the success of this model, it remains to be seen how well it can address the organizational challenges of finding better ways to recruit, train and support analysts. Nevertheless, it is a positive sign — and much better than passively awaiting 
the coming of the next maverick.

\section{Notes}

${ }^{1}$ Mandel, Applied Behavioural Science in Support of Intelligence.

${ }^{2}$ Pool, Field Evaluation in the Intelligence and Counterintelligence Context.

${ }^{3}$ Coulthart, "From Laboratory to the WMD Commission."

${ }^{4}$ Coulthart, "From Laboratory to the WMD Commission."

${ }^{5}$ United States Office of the Director of National Intelligence, Intelligence Community Directive 203.

${ }^{6}$ Heuer, Psychology of Intelligence Analysis.

${ }^{7}$ United States Office of the Director of National Intelligence, Intelligence Community Directive 203; Central Intelligence Agency, A Tradecraft Primer.

${ }^{8}$ Chang et al., "Restructuring Structured Analytic Techniques."

${ }^{9}$ Kahneman and Tversky, "Subjective Probability: A Judgment of Representativeness."

${ }^{10}$ Chang et al., "Restructuring Structured Analytic Techniques."

${ }^{11}$ Mandel and Barnes, “Accuracy of Forecasts.”; Mandel and Barnes, "Geopolitical Forecasting Skill."

${ }^{12}$ Chang and Tetlock, "Rethinking the Training of Intelligence Analysts."

${ }^{13}$ Marrin, "Training and Educating U.S. Intelligence Analysts."

${ }^{14}$ Mandel et al., "Boosting Intelligence Analysts' Judgment Accuracy."

${ }^{15}$ Dhami et al., "The 'Analysis of Competing Hypotheses' in Intelligence Analysis."

${ }^{16}$ Karvetski et al., "Improving Probability Judgment in Intelligence Analysis."

${ }^{17}$ Whitesmith, "The Efficacy of ACH."

${ }^{18}$ Mandel, "Can Decision Science Improve Intelligence Analysis?”

${ }^{19}$ Nelson, "Finding Useful Questions.”; Crupi et al., "Generalized Information Theory.”; for discussion in the context of intelligence collection, see Timms et al., "Applying Information Theory."

${ }^{20}$ Calls for increased scientific testing of analytic methods have been made, e.g., by Rieber and Thomason, "Creation of a National Institute for Analytic Methods."; Marrin, "Intelligence Analysis: Structured Methods or Intuition?"; Dhami et al., "Improving Intelligence Analysis."; and Mandel and Tetlock, "Correcting Judgment Correctives."

${ }^{21}$ Scopelliti et al., "Bias Blind Spot."

${ }^{22}$ West et al., "Cognitive Sophistication Does Not Attenuate the Bias Blind Spot."

${ }^{23}$ Heuer, email correspondence, August 15, 2010. 


\footnotetext{
${ }^{24}$ Feynman, "Cargo Cult Science."

${ }^{25}$ Nolte, "US Intelligence and its Future."

${ }^{26}$ Kerbel, "The U.S. Intelligence Community’s Kodak Moment.”

${ }^{27}$ Kent, "Estimates and Influence."

${ }^{28}$ For example, see Tetlock et al., "Forecasting Tournaments."
}

\section{Bibliography}

Chang, W., E. Berdini, D. R. Mandel, and P. E. Tetlock. "Restructuring Structured Analytic Techniques in Intelligence." Intelligence and National Security 33, no. 3 (2018): 337-356. doi:10.1080/02684527.2017.1400230

Chang, W. and P. E. Tetlock. "Rethinking the Training of Intelligence Analysts." Intelligence and National Security 31, no. 6 (2016): 903-920. doi:10.1080/02684527.2016.1147164

Coulthart, S. "From Laboratory to the WMD Commission: How Academic Research Influences Intelligence Agencies.” Intelligence and National Security 34, no. 6 (2019): 818-832. doi:10.1080/02684527.2019.1620547

Crupi, V., J. D. Nelson, B. Meder, G. Cevolani, and K. Tentori. "Generalized Information Theory Meets Human Cognition: Introducing a Unified Framework to Model Uncertainty and Information Search.” Cognitive Science 42, no. 5 (2018): 14101456. doi:10.1111/cogs.12613

Dhami, M. K., D. R. Mandel, B. A. Mellers, and P. E. Tetlock. "Improving Intelligence Analysis with Decision Science.” Perspectives on Psychological Science 10, no. 6 (2015): 753-757. doi:10.1177/1745691615598511

Dhami, M. K., I. K. Belton, and D. R. Mandel. "The 'Analysis of Competing Hypotheses' in Intelligence Analysis.” Applied Cognitive Psychology 33, no. 6 (2019): 1080-1090. doi:10.1002/acp.3550

Feynman, R. P. “Cargo Cult Science.” Engineering and Science 37, no. 7 (1974): 10-13.

Heuer, R. J., Jr. Email correspondence sent to the International Association for Intelligence Education. August 15, 2010.

Heuer, R. J., Jr. Psychology of Intelligence Analysis. Washington, DC: Central Intelligence Agency, Center for the Study of Intelligence, 1999.

Kahneman, D. and A. Tversky. "Subjective Probability: A Judgment of Representativeness.” Cognitive Psychology 3 (1972): 430-454. doi:10.1016/00100285(72)90016-3 
Karvetski, C. W., D. R. Mandel, and D. Irwin. "Improving Probability Judgment in Intelligence Analysis: From Structured Analysis to Statistical Aggregation.” Risk Analysis, in press.

Kent, S. "Estimates and Influence." In Sherman Kent and the Board of National Estimates: Collected Essays, edited by D. P. Steury, 51-59. Washington, DC: Central Intelligence Agency Centre for the Study of Intelligence, 1994.

Kerbel, J. "The U.S. Intelligence Community's Kodak Moment." The National Interest (May 15, 2014). Available from https://nationalinterest.org/feature/the-us-intelligencecommunitys-kodak-moment-10463.

Mandel, D. R. Applied Behavioural Science in Support of Intelligence: Experiences in Building a Canadian Capability. Commissioned Report to the Committee on Field Evaluation of Behavioral and Cognitive Sciences-Based Methods and Tools for Intelligence and Counter-intelligence, Division of Behavioral and Social Sciences and Education. Washington, DC: The National Academies Press, 2009.

Mandel, D. R. “Can Decision Science Improve Intelligence Analysis?” In Researching National Security Intelligence: Multidisciplinary Approaches, edited by S. Coulthart, M. Landon-Murray, and D. Van Puyvelde, 117-140. Washington, DC: Georgetown University Press, 2019.

Mandel, D. R., and A. Barnes. "Accuracy of Forecasts in Strategic Intelligence." Proceedings of the National Academy of Sciences 111, no. 30 (2014): 10984-10989. doi:10.1073/pnas.1406138111

Mandel, D. R., C. W. Karvetski, and M. K. Dhami. "Boosting Intelligence Analysts' Judgment Accuracy: What Works? What Fails?" Judgment and Decision Making 13, no. 6 (2018): 607-621. doi:10.3389/fpsyg.2018.02640

Mandel, D. R., and P. E. Tetlock. "Correcting Judgment Correctives in National Security Intelligence.” Frontiers in Psychology 9 (2018): 2640. doi:10.3389/fpsyg.2018.02640

Marrin, S. "Intelligence Analysis: Structured Methods or Intuition?" American Intelligence Journal 25, no. 1 (2007): 7-16.

Marrin, S. "Training and Educating U.S. Intelligence Analysts.” International Journal of Intelligence and CounterIntelligence 22, no. 1 (2009): 131-146. doi:10.1080/08850600802486986

Nelson, J. D. "Finding Useful Questions: On Bayesian Diagnosticity, Probability, Impact, and Information Gain.” Psychological Review 112, no. 4 (2005): 979-999. doi:10.1037/0033-295X.112.4.979 
Nolte, W. "US Intelligence and its Future: Aligning with a New and Complex Environment." Intelligence and National Security 34, no. 4 (2019): 615-618. doi:10.1080/02684527.2019.1600286

Pool, R. Field Evaluation in the Intelligence and Counterintelligence Context: Workshop Summary. Washington, DC: The National Academies Press, 2010.

Rieber, S., and N. Thomason. "Creation of a National Institute for Analytic Methods." Studies in Intelligence 49, no. 4 (2005): 71-77.

Scopelliti, I., C. K. Morewedge, E. McCormick, H. L. Min, S. Lebrecht, and K. S. Kassam. "Bias Blind Spot: Structure, Measurement, and Consequences." Management Science 61, no. 10 (2015): 2468-2486. doi:10.1287/mnsc.2014.2096

Tetlock, P. E., B. A. Mellers, N. Rorbaugh, and E. Chen. "Forecasting Tournaments: Tools for Increasing Transparency and Improving the Quality of the Debate." Current Directions in Psychological Science 23, no. 4 (2014): 290-295. doi:10.1177/0963721414534257

Timms, M., D. R. Mandel, and J. D. Nelson. "Applying Information Theory to Validate Commanders' Critical Information Requirements." In Handbook of Military and Defence Operation Research, edited by N. M. Scala and J. Howards, 331-344. Boca Raton, FL: CRC Press, 2020.

United States Office of the Director of National Intelligence. Intelligence Community Directive 203, Analytic Tradecraft. Washington, DC, 2015.

West, R. F., R. J. Meserve, and K. E. Stanovich. "Cognitive Sophistication Does Not Attenuate the Bias Blind Spot." Journal of Personality and Social Psychology 103, no. 3 (2012): 506-519. doi:10.1037/a0028857

Whitesmith, M. "The Efficacy of ACH in Mitigating Serial Position Effects and Confirmation Bias in an Intelligence Analysis Scenario." Intelligence and National Security 34, no. 2 (2019): 225-242. doi:10.1080/02684527.2018.1534640 